\title{
Numerical Wave Tank for Study Seakeeping of Amphibious Vehicle Tao Wang ${ }^{1, a}$, Xiuyue Yang $^{2}$ and Xinmin Yao ${ }^{1}$ \\ ${ }^{1}$ Department of Mechanical Engineering, Academy of Armored Force Engineering, Beijing, China \\ ${ }^{2}$ The Small Arms Demonstration \& Research Institute, Beijing, China \\ agodbless126@126.com
}

Keywords: numerical wave tank, seakeeping, amphibious vehicle

\begin{abstract}
Wave generation method and vehicle position achieving method are investigated. A numerical wave tank is made based on CFD to study seakeeping performance of amphibious vehicle. Wave is made by a board maker method and absorbed by a velocity boundary treatment. Vehicle position is achieved by solving rigid body motion equations. Moving boundary is realized by dynamic deformable grid, and free surface is captured by VOF scheme. The motion of vehicle advancing in regular head waves is simulated. Results of vehicle position and resistance indicate that simulations can achieve enough hydrodynamic characteristics to study seakeeping capacity.
\end{abstract}

\section{Introduction}

Similar studies on seakeeping are bound to ship all along. Most methods that are based on potential flow in these studies are just fitted to slim vessel. Complex shape of amphibious vehicle brings new challenge for these methods. ITTC $24^{[1]}$ points out that CFD method is a precision method to predict seakeeping capacity. CFD method is not limited by the hypothesis so it is fit for not only ship but also marine vehicle. With regard to the seakeeping behavior of an amphibious vehicle, it could be of interest to study the influence of wave on vehicle motions. The fundamental aspects of numerical simulation on seakeeping are numerical wave maker and absorber that are hot points in ocean engineering, which is crucial for study on numerical tank. For this reason, this paper discusses numerical method on wave maker and absorber and vehicle position achieving method.

\section{Numerical Wave Maker and Absorber}

Numerical Wave Tank. Numerical Wave Tank (NWT) is a generic name of numerical simulators for free surface waves, hydrodynamic forces and floating body motions ${ }^{[2]}$. Fig. 1 shows a common structure. Two important aspects should be investigated, wave maker and wave absorber.

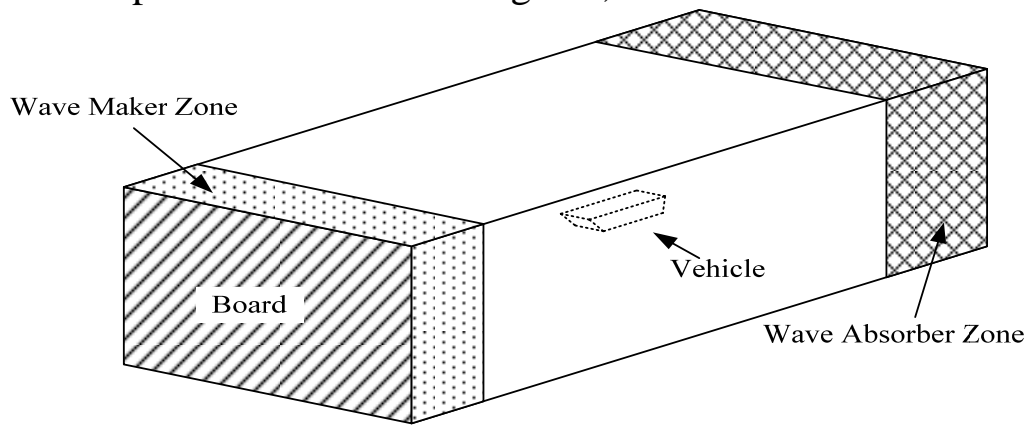

Fig. 1 Constitution of NWT

Numerical Method on Wave Maker. Wave maker is used to generate specific waves. Generic methods for this purpose involve board maker, source maker and boundary maker. Board maker is most similar to physical tank wave maker, since wave generation depends on the movement of board at the boundary of tank.

Governing equations of flow is solved in an inertial earth-fixed reference frame $(\xi, \eta, \zeta)$. At each time step, the fluid flow equations are solved, and, by integrating the elemental forces, the forces and moments acting on the vehicle are computed and projected in a non-inertial vehicle-fixed reference 
frame $(x, y, z)$, with its origin, $o$, fixed at the center of gravity of the vehicle. By the application of the rigid body equations, the translational and angular velocities of the vehicle are computed using a predictor/corrector approach.

Displacement of board is given by

$$
\xi(t)=\frac{\xi_{0}}{2} \sin \omega t
$$

where $\xi_{0}$ is displacement amplitude of board, $\omega$ is motion frequency of board.

Hence for linear wave, wave profile $\zeta$ can be given by following equation ${ }^{[3]}$

$$
\zeta=\frac{\xi_{0}}{2}\left\{\frac{4 \sinh ^{2}(c h)}{2 k h+\sinh (2 c h)} \cos (c x-\omega t)+\sum_{n=1}^{\infty} \frac{4 \sin ^{2}\left(\mu_{n} h\right)}{2 \mu_{n} h+\sinh \left(2 \mu_{n} h\right)} e^{-\mu_{n} x} \sin \omega t\right\} .
$$

where $h$ is water height of tank, $c$ is wave quantity in $2 \pi$ distance and should satisfy Eq. $3, \mu_{n}$ is given by Eq. 4 。

$$
\begin{aligned}
& c g \tanh (c h)-\omega^{2}=0 . \\
& \mu_{n} g \tan \left(\mu_{n} h\right)+\omega^{2}=0 .
\end{aligned}
$$

The second item in Eq. 2, $\sum_{n=1}^{\infty} \frac{4 \sin ^{2}\left(\mu_{n} h\right)}{2 \mu_{n} h+\sinh \left(2 \mu_{n} h\right)} e^{-\mu_{n} \xi} \sin \omega t$ will be eliminated after wave spread forward 3 times of water depth, so Eq. 2 can be simplified into

$$
\zeta=\frac{\xi_{0}}{2}\left\{\frac{4 \sinh ^{2}(c h)}{2 c h+\sinh (2 c h)} \cos (c \xi-\omega t)\right\} \text {. }
$$

$\zeta$ also satisfies $\zeta=\frac{H}{2} \cos (c \xi-\omega t)$, where $H$ is wave height. So $\xi_{0}$ can be solved by

$$
\xi_{0}=H \frac{2 c h+\sinh (2 c h)}{4 \sinh ^{2}(c h)} .
$$

Numerical Method on Wave Absorber. Wave absorber is used to eliminate the reflect wave generated from collision of incoming wave on tank wall. This paper employ the approach proposed in literature [4]. Original vertical velocity $\dot{\zeta}$ in this zone is damped to $\dot{\zeta}_{r}$ by following equation

$$
\dot{\zeta}_{r}(x, y, z, t)=\dot{\zeta}(x, y, z, t) \cdot \mu(x, z)
$$

where $\mu(x, z)$ is damping function defined by

$$
\mu(x, z)=\alpha\left(\frac{x-x_{s}}{x_{e}-x_{s}}\right)^{2}\left(\frac{z_{b}-z}{z_{b}-z_{f s}}\right) .
$$

where $(x, y, z)$ is any position in damping zone, $x_{s}$ is start position, $x_{e}$ is end position, $z_{b}$ is bottom position, $z_{f s}$ is free surface position, and $\alpha$ is a control parameter. 


\section{ALE based vehicle position achieving method}

Governing Equations of Fluid. The flow around the vehicle is turbulence since underwater part is irregular. In Euler coordinate system, unsteady viscous fluid is governed by N-S equations.

$$
\begin{aligned}
& \partial \mathbf{u} / \partial t+(\mathbf{u} \cdot \nabla) \mathbf{u}=\mathbf{f}-\rho^{-1} \nabla p+\nu \nabla^{2} \mathbf{u} . \\
& \nabla \cdot \mathbf{u}=0 .
\end{aligned}
$$

where $\nabla$ is Hamilton operator, $\mathbf{u}$ is velocity vector, $\mathbf{f}$ is body force vector, $\nabla \mathbf{p}$ is relative pressure, $v \nabla^{2} \mathbf{u}$ is diffusion term. Eq. 9 and 10 can not be used to treat moving boundary since they are only valid in undeformed grid. ALE scheme have an Euler coordinate system that can move with moving boundary at speed v. Eq. 9 can be transformed to Eq. 11 in this reference coordinate system. All variants are redefined in this coordinate system.

$$
\partial \mathbf{u} / \partial t+(\mathbf{u}-\mathbf{v}) \cdot \nabla \mathbf{u}=\mathbf{f}-\rho^{-1} \nabla p+v \nabla^{2} \mathbf{u}
$$

Basic Equations on Motion of Vehicle. Take vehicle as rigid body, i.e. not to take elasticity of vehicle into account, then the motion of vehicle can be described by Eq. 12 and 13 .

$$
\begin{aligned}
& \mathbf{m} \cdot \dot{\mathbf{v}}+\mathbf{m} \cdot(\mathbf{\Omega} \times \mathbf{v})=\int_{S} \boldsymbol{\tau} \cdot \mathbf{n} d s+\mathbf{m} \cdot \mathbf{g} \cdot \\
& \mathbf{J} \cdot \dot{\boldsymbol{\Omega}}+\mathbf{\Omega} \times(\mathbf{J} \cdot \mathbf{\Omega})=\int_{S} \mathbf{r} \times(\boldsymbol{\tau} \cdot \mathbf{n}) d s .
\end{aligned}
$$

where $\mathbf{m}$ is mass tensor, $\mathbf{v}=(u, v, w)^{T}$ is velocity vector, $\mathbf{\Omega}=(p, q, r)^{T}$ is angle velocity vector, $\mathbf{J}=\left(J_{x}\right.$, $\left.J_{y}, J_{z}\right)^{T}$ is inertia tensor, $\boldsymbol{\tau}$ is surface stress tensor, $\mathbf{n}$ is unit vector normal to $d s, \mathbf{g}$ is gravity acceleration, $\mathbf{r}$ is radius vector from ds to origin in body fixed coordinate system. $S$ is wet surface of vehicle.

Grid Updating Scheme. Dynamic deformable grid is used to drive moving boundary in simulation of flow field. Updating scheme of deformable grid should be able to build high quality mesh stably and effectively in real-time. According to trait of navigating vehicle, two schemes are used, viz. spring-based smoothing method and local remeshing method

Other Scheme. Flow field include two phases, free surface was captured by Volume of Fluid method $(\mathrm{VOF})^{[5]}$. There only one volume fraction continuity equation was need to add. Governing equations (RANS) were discreted by finite volume method, convection term was discreted by second up-wind scheme, diffusion term and source term were discreted by central scheme, Gauss-Seidel method was used to iterate the discreted equations. SIMPLE method was used to couple pressure and velocity, Euler method was used to temporal discreted.

\section{Analyses}

Regular Wave. In order to validated the above method, a target wave is simulated. Wave length is $25 \mathrm{~m}$, wave height is $0.5 \mathrm{~m}$. Fig. 2 presents the result wave pattern, and good agreement is achieved.

Wave begins to damp from $60 \mathrm{~m}$ to $75 \mathrm{~m}$ where absorber zone is located. For different wave length, absorber zone length should also be different to achieve good result. In general, length of absorber zone should be set in $1 / 2-2 / 3$ ratio of wave length.

A Vehicle Advancing in Regular Head Waves. Vehicle speed is $2 \mathrm{~m} / \mathrm{s}$ and hull length is $7 \mathrm{~m}$. Wave height is $1.4 \mathrm{~m}$, wave length is $25 \mathrm{~m}$, and wave period is $4 \mathrm{~s}$. Calm water depth in tank is $10 \mathrm{~m}$. Motion of wave maker board can be computed by Eq. 6. Computed displacement amplitude of board is $0.8 \mathrm{~m}$. Fig. 3 shows the simulated flow field. 


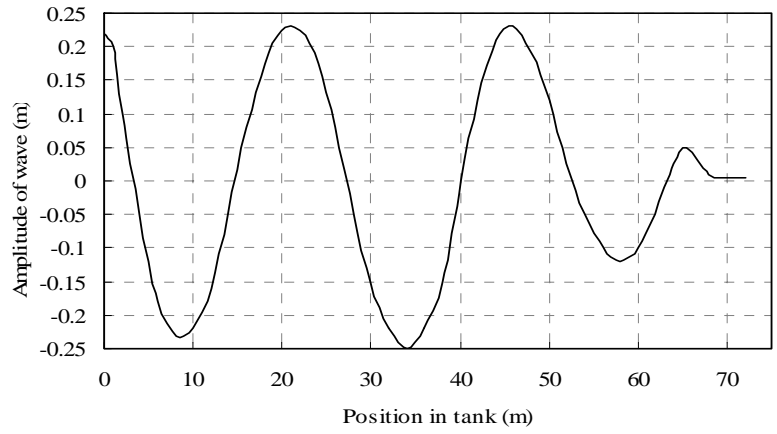

Fig.2 Simulated wave pattern

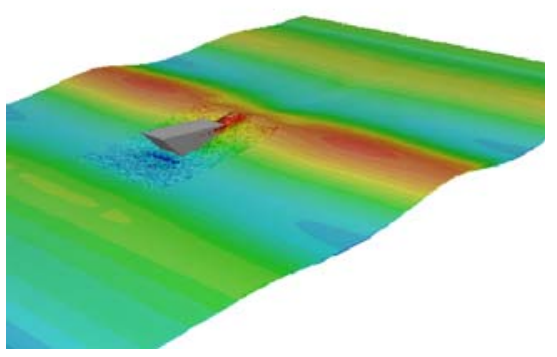

Fig.3 Simulated flow field of vehicle advancing in regular head wave

Resistance and lift is plotted in Fig. 4 and vehicle position is plotted in Fig. 5. Resistance in calm water is $10200 \mathrm{~N}$ and average resistance in head wave is $18600 \mathrm{~N}$, so average added resistance ratio is 0.82 and maximum ratio is 3 . Therefore at the same power, vehicle speed in wave will reduce to 0.55 of it in calm water. With consideration of reduction of propeller which is about 0.2 , the average reduction of speed is about 0.3 .

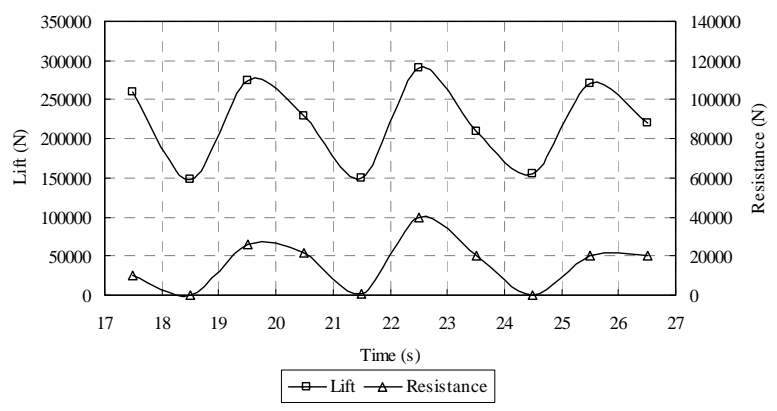

Fig.4 Lift and resistance of vehicle advancing in regular head wave

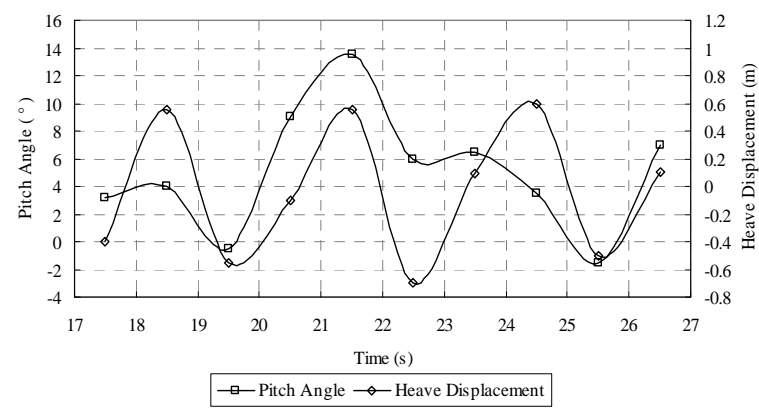

Fig.5 Position of vehicle advancing in regular head wave

\section{Conclusions}

NWT is a good choice for study seakeeping performance of amphibious vehicle. Appropriate methods on wave maker and wave absorber are key points for building NWT. In this paper, Wave is made by a board maker method and absorbed by a velocity boundary treatment. Vehicle position is achieved by solving rigid body motion equations. The motion of vehicle advancing in regular head waves is simulated. Results of vehicle position and resistance indicate that simulations can achieve enough hydrodynamic characteristics to study seakeeping capacity.

\section{References}

[1] Technical Committees of the 24th ITTC. Final report of the resistance committee [EB/OL]. http://www.ittc.ncl. ac.uk/reports/index.htm

[2] Katsuji TANIZAWA. The State of the Art on Numerical Wave Tank. Proceeding of 4th Osaka Colloquium on Seakeeping Performance of Ships,Osaka, Japan, (2000), p. 95-114

[3] U R SELLF, DEAN R G, YU Y S. Forced small amplitude water waves: acomparison of theory and experiment. J Fluid Mech, Vol. 7(1960), p. 33-52

[4] ARA IM, PAUL U K, CHENG L Y. A technique for open boundary treatment in numerical wave tanks. J Soc Naval Archit Japan, Vol. 173(1993), p. 45-50

[5] HIRT C W, NICHOLS B D. Volume of flu id(VOF ) method for the dynamics of free boundaries. J Comput Phys, Vol. 39(1981), p. 201-225 